\title{
Manifest der Ärzte «Gesundheitspolitik: Stopp der Selbstversenkung»: bereits mehr als 1000 Unterschriften
}

1042 Ärzte haben das nachfolgende Manifest, das von einem tiefen und weitverbreiteten Unbehagen zeugt, unterschrieben. Sie erachten es als ihre Pflicht, die Bevölkerung zu alarmieren. Sie sind über die Auswirkungen der derzeitigen politischen Massnahmen oder Projekte besorgt, welche die Qualität der Medizin sowie den Zugang zur Pflege für alle bedrohen.

Die unterzeichnenden Ärzte behaupten nicht, sie hätten fixfertige Antworten auf die derzeitigen Probleme. Sie fordern jedoch, dass die Grundlagen der Überlegungen neu definiert und dass endlich die richtigen Fragen gestellt werden. Insbesondere halten sie es für notwendig, dass man sich zuerst Fragen über die wirklichen Gesundheitsbedürfnisse der Bevölkerung stellt und über den Preis, den diese bereit ist, dafür zu bezahlen.

Die ersten 1042 Unterschriften sowie das Manifest wurden in der Nummer 2502 von «Médecine et Hygiène» veröffentlicht (27. Oktober 2004, http://revue.medhyg.ch/manifeste/signatures/).

Damit unserem Anliegen wirklich Beachtung geschenkt wird, brauchen wir ein Maximum an Unterschriften. Unterschreiben Sie und geben Sie dieses Manifest zur Unterschrift weiter! Die weiteren Unterschriften werden in einer nächsten Ausgabe von «Médecine et Hygiène» veröffentlicht.

Um zu unterschreiben, brauchen Sie nur Ihre persönlichen Angaben auf der Internetseite einzugeben: http://revue.medhyg.ch/manifeste/de/ (Achtung: kein www!).

Ausserdem ist Ihre Hilfe notwendig, damit wir diese Aktion weiterverfolgen können. Im besonderen, um dieses Manifest in verschiedenen Zeitschriften zu veröffentlichen. Wir danken Ihnen im voraus für Ihren Einsatz und Ihre finanzielle Unterstützung: BCV, 1002 Lausanne. Postscheckkonto 10-725-4, Clearing 767, Konto 50929944 «Manifeste des Médecins Suisses».

\section{Manifest}

Gesundheitspolitik: Stopp der Selbstversenkung Wir, Ärzte aller Generationen und Fachrichtungen, als Zeugen menschlichen Leidens und als Behandelnde, machen die Bevölkerung auf die Konsequenzen der aktuellen Schweizerischen Gesundheitspolitik aufmerksam.

Die gegenwärtigen Reformvorhaben stellen eine substantielle Bedrohung für die Medizin dar - nicht nur für die Qualität der Behandlung und
Pflege, sondern auch für die ethischen Grundlagen. Diese Vorhaben sind nicht nur unzusammenhängend, ohne wissenschaftlichen Bezug und ökonomisch haltlos, sie torpedieren auch die Grundwerte der Gesellschaft und unserer Arbeit, d.h. die menschliche Würde, die Intimsphäre, das Eingehen auf unterschiedliche Bedürfnisse sowie das Recht auf freien Zugang zu einer qualitativ hochwertigen medizinischen Versorgung. Sie stellen auch gewaltsam unsere Identität als Behandelnde in Frage.

Angesichts dieser ernsten Situation haben wir beschlossen, nicht mehr länger zu schweigen und entschieden gegen die Reformvorhaben einzutreten. Auch wir stimmen einer Weiterentwicklung des Gesundheitswesens zu, aber unter Einbezug aller Betroffenen. Gemeinsam mit den Patienten und dem Pflegepersonal wollen wir uns aktiv in die laufende Diskussion einbringen. Die demokratische Entwicklung der Medizin ist nur gewährleistet, wenn unser aller Wissen und die Rahmenbedingungen zur Berufsausübung eingebracht werden können. Wir lehnen die aktuell fehlende Diskussionsbereitschaft und den fehlenden Meinungsaustausch $\mathrm{ab}$ und prangern die autoritäre Verachtung an, welche diejenigen, die die politische und ökonomische Macht ausüben, an den Tag legen.

Wir fordern eine Kurskorrektur, nämlich vom ineffizienten und zerstörerischen Autoritarismus weg hin zum partnerschaftlichen Zusammenarbeiten. Dabei wollen wir unserer Verantwortung als Mediziner gerecht werden. Das heisst, dass wir uns nicht nur auf die Ausführung von Vorgaben reduzieren lassen, sondern unter Achtung unserer Kompetenzen aktiv an der Entwicklung des Schweizerischen Gesundheitswesens beteiligt sein wollen.

Die Ausgestaltung unseres Gesundheitswesens darf nicht allein den Wirtschaftsexperten überlassen werden! Wir Ärzte sind bereit, uns den Herausforderungen zu stellen und bieten den Regierungsbehörden unsere Zusammenarbeit an. Dies in der Überzeugung, dass es uns nur gemeinsam gelingt, den richtigen Weg in Richtung einer effizienten, kostenbewussten und menschlichen Gesundheitsversorgung in der Schweiz zu finden.

Pierre de Vevey, Chavornay; Bertrand Kiefer, Genève; Alain Frei, Lausanne; Bernard Giorgis, Romanel; Jacqueline Landolt Jaquet, Morges; François Pilet, Vouvry; Oscar Matzinger, Lausanne.
E-Mail:

Bertrand.Kiefer@medhyg.ch 